\title{
Urban particulate matter triggers lung inflammation via the ROS- MAPK-NF-kB signaling pathway
}

\author{
Jian Wang*, Jianan Huang*, Linlin Wang*, Cuicui Chen, Dong Yang, Meiling Jin, Chunxue Bai, Yuanlin \\ Song
}

Department of Pulmonary Medicine, Zhongshan Hospital, Fudan University, Shanghai 200030, China

Contributions: (I) Conception and design: Y Song, J Wang; (II) Administrative support: Y Song, C Bai; (III) Provision of study materials or patients: D Yang, M Jin; (IV) Collection and assembly of data: J Huang, C Chen, L Wang; (V) Data analysis and interpretation: J Wang, J Huang; (VI) Manuscript writing: All authors; (VII) Final approval of manuscript: All authors.

*These authors contributed equally to this work.

Correspondence to: Yuanlin Song and Chunxue Bai. Department of Pulmonary Medicine, Zhongshan Hospital, Fudan University, Shanghai 200030,

China. Email: song.yuanlin@zs-hospital.sh.cn; bai.chunxue@zs-hospital.sh.cn.

Background: Particulate matter (PM) is a high risk factor for various respiratory diseases and triggers an inflammatory response in lung tissues. However, the molecular mechanism of the PM-induced inflammatory response is incompletely understood.

Methods: Human bronchial epithelial cells (HBECs) were treated with the urban PM 1649b for assessment of the inflammatory response. The intracellular level of reactive oxygen species (ROS) was measured by flow cytometry. PM-activated signaling pathways were addressed with specific inhibitors. In vivo, the C57 mice model of PM-induced acute lung inflammation was established with intratracheal instillation of PM for 2 consecutive days. The oxidant stress in lung tissues was assessed with dihydroethidium (DHE) staining, and malondialdehyde (MDA) activity and hydrogen peroxide $\left(\mathrm{H}_{2} \mathrm{O}_{2}\right)$ assays. The histopathologic changes in lung tissues and number of inflammatory cells in bronchoalveolar lavage fluid (BALF) were examined. Expression of pro-inflammatory cytokines in BALF was measured by ELISA.

Results: PM increased the expression of interleukin (IL)-1 $\beta$, IL-6, IL-8, matrix metalloproteinase (MMP)9 and cyclooxygenase (COX)-2 in a dose-dependent manner. ROS generation and activation of MAPK (ERK, JNK, p38 MAPK) and NF-кB pathways were detected in PM-exposed HBECs. Pretreatment with $\mathrm{N}$-acetylcysteine (NAC) led to the inflammatory response, ROS level and activation of the MAPK and NF- $\kappa$ B pathways to be attenuated. Blockade of ERK, JNK or p38 MAPK pathway with specific inhibitor prevented the release of pro-inflammatory cytokines and activation of the NF- $\kappa \mathrm{B}$ pathway. Inhibition of the NF-кB pathway reduced the expression of pro-inflammatory cytokines. In vivo, PM exposure increased oxidant stress in lung tissues, infiltration of inflammatory cells around PM in lung tissues, the number of total cells and inflammatory cells in BALF, and the concentrations of IL-1 $\beta$, IL-6, IL-8 and MMP-9 in BALF, all of which were reversed partially upon NAC treatment.

Conclusions: PM exposure enhanced the airway inflammatory response significantly through ROSmediated activation of MAPK (ERK, JNK, p38 MAPK) and downstream NF- $\kappa$ B signaling pathways. Oxidative stress appeared to be the key regulator for PM-induced lung inflammation. These results suggested the molecular mechanism of lung inflammation caused by PM.

Keywords: Inflammation; lung; oxidative stress; particulate matter (PM); signaling pathway

Submitted May 17, 2017. Accepted for publication Sep 18, 2017.

doi: $10.21037 /$ jtd.2017.09.135

View this article at: http://dx.doi.org/10.21037/jtd.2017.09.135 


\section{Introduction}

Air pollution has become an increasing threat to public health, particularly in urban areas. In 2016, the World Health Organization (WHO) reported that $>80 \%$ of urban residents lived in places where WHO guidelines for air quality levels were not met (1). It has been estimated that urban air pollution contributes to approximately 1.3 million deaths worldwide per year (2). Particulate matter (PM) is the major air-polluting agent in urban areas worldwide. Epidemiologic data have shown that PM exposure has a high correlation with bronchial asthma (3), chronic obstructive pulmonary disease $(4,5)$, lung cancer (6), atherosclerosis (7), ischemic stroke (8), congestive heart failure (9), myocardial ischemia (10) and birth defects (11).

The main sources of urban PM are road traffic, industrial emissions, construction processes, fuel combustion, resuspension of soil, and secondary emissions (12). The aerodynamic diameter and components of PM affect its biologic effects on humans, but vary with regard to sources, time and location. With respect to aerodynamic diameter, PM can be classified as "PM10" $(\leq 10 \mu \mathrm{m})$, "PM2.5" ( $\leq 2.5 \mu \mathrm{m})$ and "PM0.1" $(\leq 0.1 \mu \mathrm{m})$. The lungs are the first targets to be affected by air pollutants. PM2.5 and PM0.1 can be inhaled into small airways and alveoli, and even absorbed into blood to further damage other organs or tissues, but PM10 deposits only in large airways (7). The components of PM in air are complicated and vary according to influencing factors (13-15). Common components are sulfates, nitrates, ammonium, acids, heavy metals (including lead, copper, manganese, nickel, cadmium and titanium), polycyclic aromatic hydrocarbons (PAHs), crustal material, elemental and organic carbon, and biologic materials (e.g., allergens and microbial compounds) (16). However, the association between specific chemical components of PM in air and adverse human health outcomes is not clear.

Ambient exposure to PM can cause various biologic effects on different cell types in the lungs. The polarization of macrophages is induced by PM and the expression of several reactive oxygen species (ROS)-mediated pro-inflammatory cytokines (e.g., interleukin (IL)-6, IL-1 $\beta$, tumor necrosis factor (TNF)- $\alpha$ and granulocytemacrophage colony-stimulating factor) is increased (17). PM exposure also induces the apoptosis and proinflammatory response of airway epithelial cells via complicated signaling networks (18). In addition, PM induces epithelial-to-mesenchymal transition, which can result in cancer development and pulmonary fibrosis (19). Recently, many studies have focused on investigating the key components of PM that determine their biological effects. The metal contents or organic components of PM can lead to the release of pro-inflammatory cytokines via activation of oxidative stress (20). Oxidative stress is caused by an imbalance between oxidation and anti-oxidation to produce excessive ROS. Oxidative stress acts an important mediator in the pathology of various PM-induced human diseases (21). Furthermore, several signaling pathways, including toll-like receptors, mitogen-activated protein kinase (MAPK), phosphatidylinositol 3-kinase and nuclear erythroid 2-related factor 2, are activated by PM to regulate its biologic effects (18). However, the relationship between the specific components of PM and signaling pathways is not known.

The constituents of the reference urban dust material SRM $1649 b$ (which is usually abbreviated to "1649b") were identified for the present study. $1649 \mathrm{~b}$ consisted mainly of PAHs, polychlorinated biphenyl congeners, pesticides, and dioxins, all of which are the main components of urban PM. However, the evidence that can clarify the molecular mechanism linking $1649 \mathrm{~b}$ exposure to the regulation of inflammatory response is lacking. Here, we developed a human bronchial epithelial cell (HBEC) model to reveal the specific signaling pathway participating in a $1649 \mathrm{~b}$-induced inflammatory response. Furthermore, we established an in vivo model to demonstrate the key regulator of oxidative stress for $1649 \mathrm{~b}$-induced lung inflammation.

\section{Methods}

\section{Reagents and antibodies}

$1649 \mathrm{~b}$ was purchased from NIST (Gaithersburg, MD, USA). N-acetylcysteine (NAC), SP600125 and 2',7'-dichlorofluorescin diacetate (DCFDA) were purchased from Beyotime (Shanghai, China). Dihydroethidium (DHE) was obtained from Sigma-Aldrich (Saint Louis, MO, USA). U0126, SB203580 and BAY 11-7082 were obtained from Selleck (Houston, Texas, USA). Antibodies against cyclooxygenase (COX)-2, phospho-ERK, ERK, phosphoJNK, JNK, phospho-p38 MAPK, p38 MAPK, phospho$\mathrm{NF}-\kappa \mathrm{B}$ p 65 , and NF- $\mathrm{B}$ p65 were purchased from Cell Signaling Technology (Danvers, MA, USA). Antibodies against GAPDH were purchased from Beyotime (Shanghai, China). The mRNA primers were synthesized by Synbio Technologies (Suzhou, China). The reagents used for real- 
Table 1 Primers used in the study

\begin{tabular}{llc}
\hline Genes & \multicolumn{1}{c}{ Forward } & Reverse \\
\hline GAPDH & 5'-CCACCCATGGCAAATTCCATGGCA-3' & 5'-TCTACACGGCAGGTCAGGTCCACC-3' \\
IL-1 $\beta$ & 5'-TGGCAATGAGGATGACTTGT-3' & 5'-TGGTGGTCGGAGATTCGTA-3' \\
IL-6 & 5'-TTCGGTCCAGTTGCCTTCT-3' & 5'-GGTGAGTGGCTGTCTGTGTG-3' \\
MMP-9 & 5'-GCTGGCAGAGGAATACCTGTAC-3' & 5'- CAGGGACAGTTGCTTCTGGA-3' \\
COX-2 & 5'-TGAGTGTGGGATTTGACCAG-3' & 5'-TGTGTTTGGAGTGGGTTTCA-3' \\
IL-8 & 5'-TTGCCAAGGAGTGCTAAAGAA-3' & 5'-GCCCTCTTCAAAAACTTCTCC-3' \\
\hline
\end{tabular}

time PCR reaction and cDNA synthesis kits were purchased from Takara Bio (Shiga, Japan). The reagents used for western blotting were obtained from Beyotime.

\section{Cells and animals}

HBECs were purchased from the Chinese Academy of Sciences (Shanghai, China) and cultured in RPMI-1640 medium (Hyclone, Logan, UT, USA) supplemented with $10 \%$ fetal bovine serum (Gibco, Waltham, MA, USA) and $50 \mathrm{U} / \mathrm{mL}$ penicillin and streptomycin (Gibco) at $37{ }^{\circ} \mathrm{C}$ in a $5 \% \mathrm{CO}_{2}$ culture chamber. Male C57 mice (6-8 weeks, 20-22 g) were purchased from SLAC Laboratory Animals (Shanghai, China). All protocols for animal experiments were approved by the Animal Care and Use Committee of Zhongshan Hospital, Fudan University.

\section{Research design}

For in vitro experiments, $\mathrm{PM}$ was suspended in $\mathrm{PBS}$ at a stock concentration of $4 \mathrm{mg} / \mathrm{cm}^{3}$ and HBECs were treated with $\mathrm{PM}$ at different concentrations. Inhibitor NAC (5 mM), U0126 (10 $\mu \mathrm{M})$, SP600125 (10 $\mu \mathrm{M})$, SB203580 $(10 \mu \mathrm{M})$ or BAY 11-7082 (5 $\mu \mathrm{M})$ was used to treat HBECs 30 min before PM stimulation. For in vivo experiments, mice were treated with $100 \mu \mathrm{g}$ PM in $25 \mu \mathrm{L}$ PBS per day by intratracheal instillation for 2 consecutive days to establish a PM-induced model of acute lung inflammation, as reported previously (22). NAC (200 mg/kg, i.p.) was injected $1 \mathrm{~h}$ before PM stimulation every day.

\section{Real-time PCR}

TRIzol $^{\text {TM }}$ (Invitrogen, Carlsbad, USA) was used to lyse cells. RNA extraction and reverse transcription were performed according to manufacturer instructions. Quantitative real-time PCR was done using SYBR® Premix Ex Taq ${ }^{\text {TM }}$ (TaKaRa Bio) on a real-time PCR system (Bio-Rad Laboratories, Hercules, CA, USA). The primer sequences are shown in Table 1. The relative level of mRNA expression of each target gene is shown as a $2^{-\Delta \Delta C t}$ value.

\section{Western blotting}

Total protein of cells from different groups was obtained using RIPA buffer containing phenylmethanesulfonyl fluoride (PMSF, Beyotime) and phosphatase inhibitors (Biotool, Houston, TX, USA). The protein concentration was measured with a BCA Protein Assay kit (Beyotime). An identical amount of lysates $(30 \mu \mathrm{g})$ underwent SDSPAGE and was then transferred to a PVDF membranes. The latter were blocked with blocking buffer for $1 \mathrm{~h}$ and then incubated with different primary antibodies (1:1,000 dilution) overnight at $4{ }^{\circ} \mathrm{C}$. After washing thrice in TBST, the PVDF membranes were incubated with anti-mouse or anti-rabbit horseradish peroxidase-conjugated secondary antibodies (1:5,000 dilution) for $1 \mathrm{~h}$ at room temperature. Immunoreactive bands were detected by ECL reagents (Thermo Scientific, Waltham, MA, USA) on A Bio-Rad Laboratories system. The intensity of protein bands was quantified by AlphaEaseFC v4.0.

\section{ROS measurement}

After different treatments, HBECs were loaded with DCFDA $(10 \mu \mathrm{M})$ in serum-free 1,640 medium for $20 \mathrm{~min}$ at $37{ }^{\circ} \mathrm{C}$ in a $5 \% \mathrm{CO}_{2}$ culture chamber. Then HBECs were washed with PBS thrice and digested with trypsin-EDTA solution (Gibco). ROS quantitation was measured by flow cytometry (Aria III; Becton Dickinson, San Jose, CA, USA) 
based on fluorescence intensity. For in vivo studies, fresh lung tissues were embedded in OCT and sliced and stained with DHE for ROS detection, as described previously (23). DHE was oxidized by superoxide to emit red fluorescence which was detected by a fluorescence microscope (Olympus, Tokyo, Japan). The relative fluorescence intensity was analyzed by Image J v1.4.3.67.

\section{Malondialdebyde (MDA) activity and bydrogen peroxide $\left(\mathrm{H}_{2} \mathrm{O}_{2}\right)$ assay}

Levels of MDA and $\mathrm{H}_{2} \mathrm{O}_{2}$ in lung tissues were detected using a lipid peroxidation MDA assay kit and $\mathrm{H}_{2} \mathrm{O}_{2}$ assay kit (Beyotime) according to manufacturer' instructions, respectively. Briefly, fresh lung tissues were lysed with RIPA and then centrifuged at $12,000 \mathrm{~g}$ for $10 \mathrm{~min}$ at $4{ }^{\circ} \mathrm{C}$. The supernatant was harvested for the measurement of the MDA level and protein concentrations. Then the MDA level was normalized with the protein concentration. Similarly, fresh lung tissues were lysed and then centrifuged at $12,000 \mathrm{~g}$ for $5 \mathrm{~min}$ at $4{ }^{\circ} \mathrm{C}$. The supernatant was collected for the measurement of $\mathrm{H}_{2} \mathrm{O}_{2}$ and protein concentration. Then, the $\mathrm{H}_{2} \mathrm{O}_{2}$ level was normalized according to the protein concentration.

\section{Histopathologic analyses}

Lungs from different groups were harvested and fixed in $4 \%$ paraformaldehyde. Then, lungs were embedded in paraffin and sliced and stained with hematoxylin and eosin (H\&E) according to manufacturer' instructions. Pathologic changes were imaged and scored as reported previously (24).

\section{Bronchoalveolar lavage fluid (BALF) collection and inflammatory cell counts}

BALF was collected according to methods detailed previously. Briefly, mice were anesthetized with avertin (25 mg/kg body weight, Sigma-Aldrich). Tracheas were dissociated and cannulated. Lungs were lavaged with $1 \mathrm{~mL}$ PBS thrice to obtain BALF. The latter was centrifuged at $800 \mathrm{~g}$ for $5 \mathrm{~min}$ at room temperature. Then supernatant was collected and stored at $-80^{\circ} \mathrm{C}$ for further analyses. Cell pellets were resuspended with $0.5 \mathrm{~mL}$ of PBS and the total cell number was counted. Then, $100 \mu \mathrm{L}$ of the resuspended cells was spun to the slide and air-dried and then fixed with 4\% paraformaldehyde overnight. Cells were stained with $\mathrm{H} \& \mathrm{E}$ and the number of macrophages and neutrophils in 200 cells was counted. The data were shown as $10^{5} / \mathrm{mL}$ BALF.

\section{Enzyme-linked immunosorbent assay (ELISA)}

The concentration of IL-1 $\beta$, IL-6, IL-8 and MMP-9 in BALF was measured using ELISA kits (4A Biotech, Beijing, China) according to manufacturer' protocols.

\section{Statistical analyses}

SPSS v19.0 (IBM, Armonk, NY, USA) was used for statistical analyses. Results were the mean \pm SEM. Differences between groups were addressed using one-way analysis of variance. $\mathrm{P}<0.05$ was considered significant.

\section{Results}

\section{PM induces an inflammatory response in HBECs}

To investigate the pro-inflammatory effect of $1649 \mathrm{~b}$ on HBECs, the latter were stimulated with 100,300 or $500 \mu \mathrm{g} / \mathrm{cm}^{3}$ PM for $24 \mathrm{~h}$, respectively. The mRNA levels of pro-inflammatory cytokines such as IL-1 $1 \beta$, IL-6 and IL-8 were increased significantly in a dose-dependent manner (Figure 1A). MMPs play important parts in the pathologic processes of several respiratory diseases by causing tissue remodeling (25). Thus, MMP-9 expression was measured upon PM treatment. The mRNA level of MMP-9 was also increased in a dose-dependent manner (Figure 1A). COX-2 expression is induced by several inflammatory stimuli and COX-2 can regulate inflammatory processes in different cell types (26). Exposure of HBECs to PM increased COX-2 expression in a dose-dependent manner at gene and protein levels (Figure 1B-D). These results suggested that PM exposure could promote an inflammatory response in HBECs.

\section{PM promotes ROS production in HBECs}

Accumulating evidence suggests that ROS plays an important part in the inflammatory response of PM in different cell types (27). Here, HBECs were stimulated with PM at different doses and times to determine the level of ROS generation. When HBECs were treated with different concentrations of PM for $24 \mathrm{~h}, \mathrm{PM}$ enhanced the ROS production significantly in a dose-dependent manner according to flow cytometry (Figure 2A). Furthermore, HBECs were exposed to $300 \mu \mathrm{g} / \mathrm{cm}^{3}$ PM for different time periods and the level of ROS generation was enhanced in a time-dependent manner (Figure 2B). There was a distinct increase in ROS levels in HBECs $1 \mathrm{~h}$ after PM treatment. These data suggested that ROS was increased significantly at the early stage of PM exposure, and might be an 


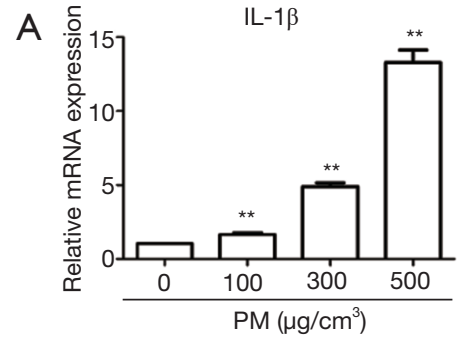

B

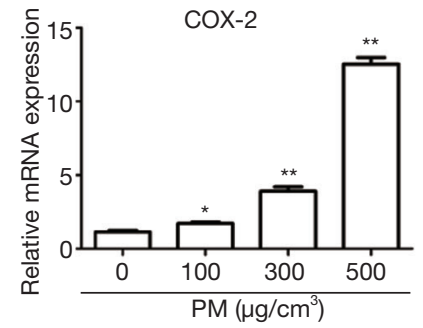

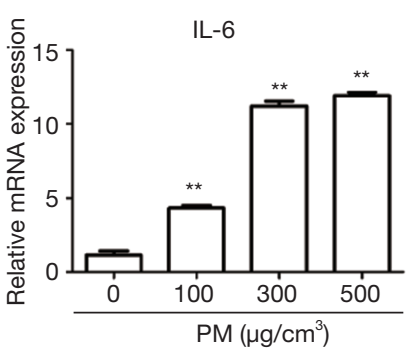

C

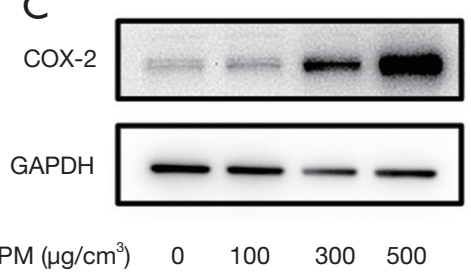

IL-8

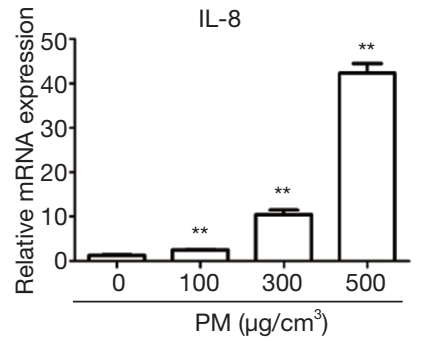

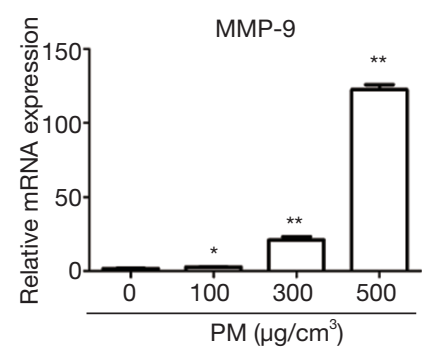

$\mathrm{D}$

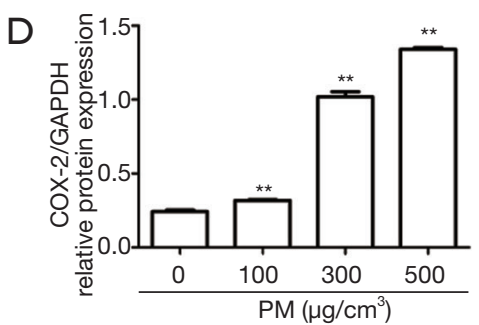

Figure 1 PM induces the inflammatory response in HBECs. (A,B) HBECs were treated with PM in a dose-dependent manner (100, 300 and $500 \mu \mathrm{g} / \mathrm{cm}^{3}$ ) for $24 \mathrm{~h}$ and mRNA expression of IL-1 $\beta$, IL-6, IL-8, MMP-9 and COX-2 were detected by RT-PCR. (C) Western blotting was used to detect the expression of COX-2 protein in HBECs upon PM treatment. Compared with GAPDH expression, the optical densities of protein' bands are shown in (D). Values are the mean $\pm \mathrm{SEM}$;, $\mathrm{P}<0.05$ or ** $\mathrm{P}<0.01$, compared with the control group; $\mathrm{n}=3$. HBEC, human bronchial epithelial cell; PM, particulate matter.

important mediator in the PM-induced inflammatory response.

To further clarify the role of oxidative stress in the PMinduced inflammatory response, HBECs were treated with the antioxidant NAC. Flow cytometry showed NAC (0.5 or $5 \mathrm{mM}$ ) could reduce $\mathrm{PM}$-induced ROS generation significantly (Figure 2C). NAC attenuated PM-induced COX-2 expression in HBECs at gene and protein levels (Figure 2D-F). Moreover, the expression of IL-1 $\beta$, IL-6, IL-8 and MMP-9 was inhibited significantly by pretreatment with NAC in PM-stimulated HBECs (Figure 2G). These results showed the key role of ROS in the PM-induced inflammatory response.

\section{The MAPK patbway is involved in the PM-induced inflammatory response}

The MAPK pathway can be divided into ERK, JNK and p38 MAPK pathways, which have a close association with inflammation, cellular growth and stress response (28). To define activation of the MAPK pathway upon PM treatment, western blotting was carried out. We showed that PM could activate the ERK, JNK, and p38 MAPK pathways in a timedependent manner by measuring the phosphorylation of ERK, JNK, and p38 MAPK, respectively (Figure $3 A, B$ ). Expression of p-ERK, p-JNK, and p-p38 MAPK reached a peak $1 \mathrm{~h}$ after $\mathrm{PM}$ exposure and declined thereafter. In addition, pretreatment with NAC blocked PM-induced activation of ERK, JNK, and p38 MAPK pathways in HBECs (Figure 3C,D). These results suggested that MAPK activation was mediated by ROS and might be implicated in the PM-induced inflammatory response in HBECs.

To clarify the role of the MAPK pathway in the PMinduced inflammatory response, an ERK inhibitor (U0126), JNK inhibitor (SP600125), and p38 MAPK inhibitor (SB203580) were used, respectively. Pretreatment with U0126, SP600125 or SB203580 could significantly attenuate PM-induced expression of IL-1 $\beta$ and MMP-9. Compared with the PM treatment group, IL-6 expression was inhibited by pretreatment with U0126 or SB203580, whereas IL-8 expression was inhibited only by pretreatment with U0126 or SP600125, respectively (Figure 4A-C). In addition, PM-induced COX-2 expression was also inhibited significantly at gene and protein levels by pretreatment with U0126, SP600125 or SB203580, respectively (Figure 4A-I). Taken together, these results suggested that ROS-mediated MAPK pathway played an important part in the PMinduced inflammatory response.

\section{Activation of NF- $\kappa B$ patbway regulates the PM-induced inflammatory response}

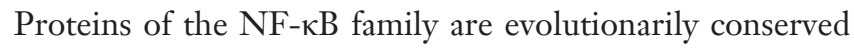


A

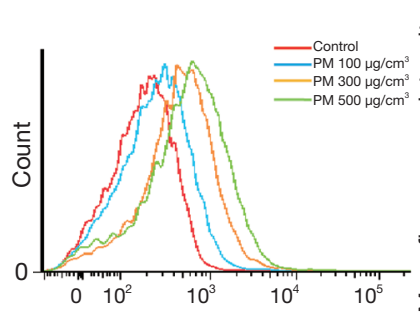

C

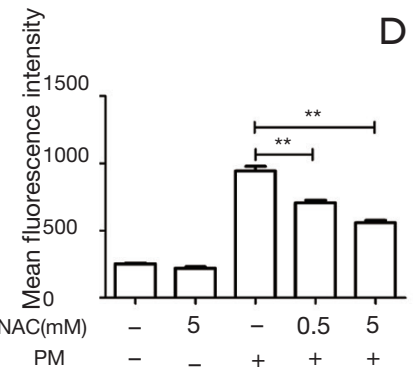

G

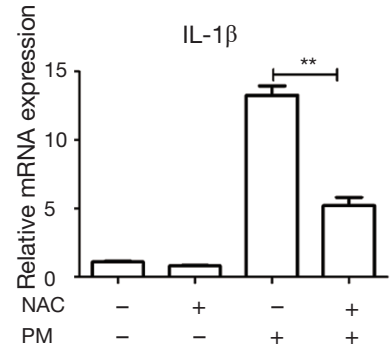

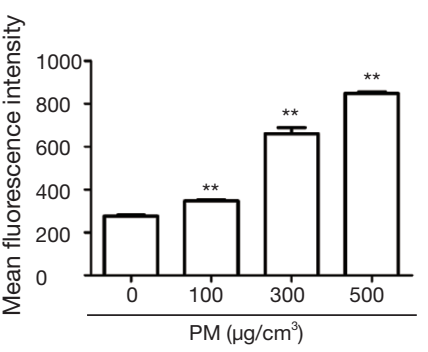
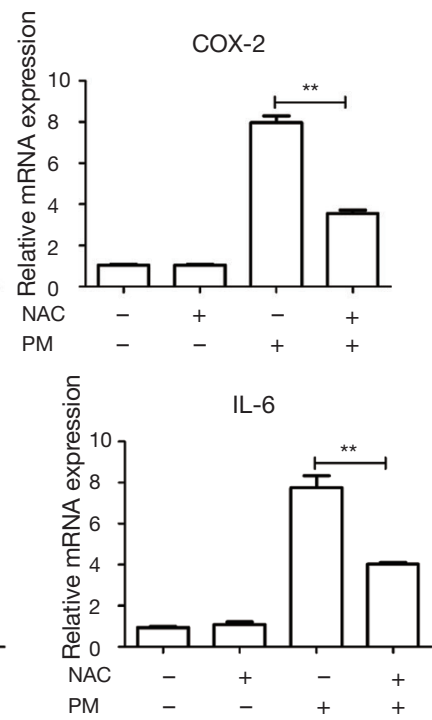

B

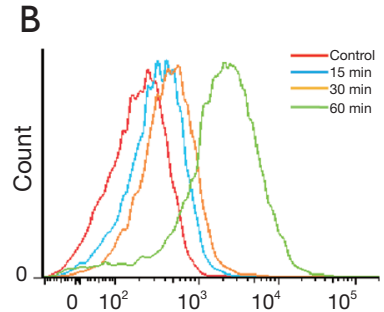

$E$
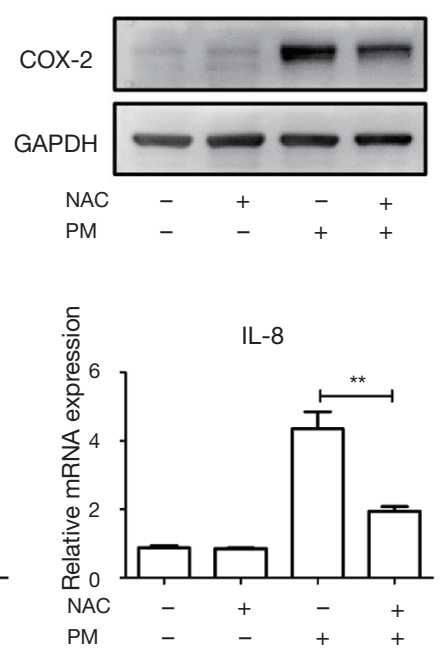

$\mathrm{F}$
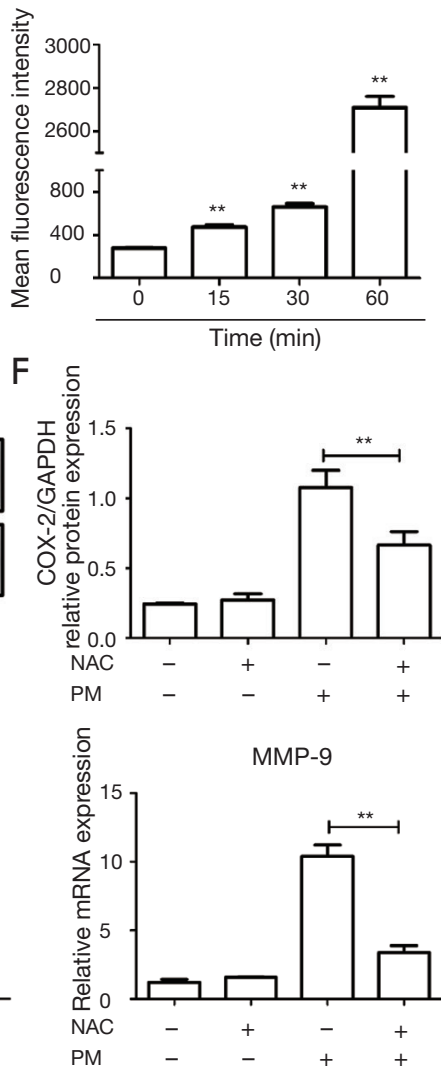

Figure 2 Role of oxidative stress in the PM-induced inflammatory response in HBECs. (A,B) HBECs were treated with PM in a dosedependent $\left(100,300\right.$ and $\left.500 \mu \mathrm{g} / \mathrm{cm}^{3}\right)$ manner for $24 \mathrm{~h}$ or treated with $300 \mu \mathrm{g} / \mathrm{cm}^{3} \mathrm{PM}$ in a time-dependent $(15,30$ and $60 \mathrm{~min}) \mathrm{manner}$. The ROS level was detected and quantified by flow cytometry. Values are the mean $\pm \mathrm{SEM}$; ${ }^{* *}, \mathrm{P}<0.01$, compared with the control group; $\mathrm{n}=6$. (C) HBECs were pretreated with NAC ( 0.5 or $5 \mathrm{mM})$ for $30 \mathrm{~min}$ and then stimulated with $300 \mu \mathrm{g} / \mathrm{cm}^{3} \mathrm{PM}$. ROS generation was quantified by flow cytometry. Values are the mean $\pm \mathrm{SEM}$; ${ }^{* *}, \mathrm{P}<0.01$, compared with the PM group; $\mathrm{n}=6$. (D) HBECs were pretreated with $5 \mathrm{mM}$ NAC before PM stimulation. The mRNA level of COX-2 was detected by RT-PCR. Values are the mean $\pm \mathrm{SEM}$; ** $\mathrm{P}<0.01$, compared with the PM group; $\mathrm{n}=3$. (E) Western blotting was used to detect the expression of COX-2 protein in NAC-pretreated HBECs upon PM stimulation. Compared with GAPDH expression, the optical densities of protein' bands are shown in (F). Values are the mean \pm SEM; ${ }^{* *}, \mathrm{P}<0.01$, compared with the PM group; $\mathrm{n}=3$. (G) HBECs were pretreated with $5 \mathrm{mM}$ NAC before PM stimulation. The mRNA level of IL-1 $\beta$, IL-6, IL-8 and MMP-9 was detected by RT-PCR. Values are the mean \pm SEM; **, $\mathrm{P}<0.01$, compared with the PM group; $\mathrm{n}=3$. HBEC, human bronchial epithelial cell; NAC, N-acetylcysteine; PM, particulate matter.

mediators which respond to different stress stimuli to modulate cell growth, cell differentiation, immunity, and inflammation (29). Studies have indicated that metalcontaining $\mathrm{PM}$ can activate the NF- $\mathrm{\kappa B}$ pathway in different cell types (18). To investigate activation of a PM-

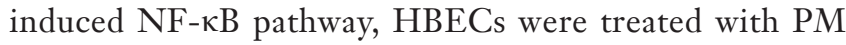
for different times. Western blotting showed that PM could increase activation of the NF- $\mathrm{KB}$ pathway in a timedependent manner. Expression of p-p65 reached a peak $1 \mathrm{~h}$ after PM exposure and declined thereafter (Figure $5 A, B$ ). Pretreatment with NAC could inhibit activation of the NF- $\mathrm{KB}$ pathway significantly (Figure $5 C, D$ ). In addition, the NF- $\mathrm{KB}$ pathway could be blocked by pretreatment with U0126, SP600125 or SB203580 in PM-treated HBECs, respectively (Figure $5 E, F$ ). These results suggested that the $\mathrm{NF}-\kappa \mathrm{B}$ pathway was mediated by ROS and MAPK in the PMinduced inflammatory response.

To clarify the role of the NF- $\kappa \mathrm{B}$ pathway in the PMinduced inflammatory response, an inhibitor of NF- $\mathrm{KB}$ pathway, Bay 11-7082, was used. Compared with the PMtreatment group, pretreatment with Bay 11-7082 could attenuate PM-induced expression of IL-1 $\beta$, IL-6, IL-8 and 
A

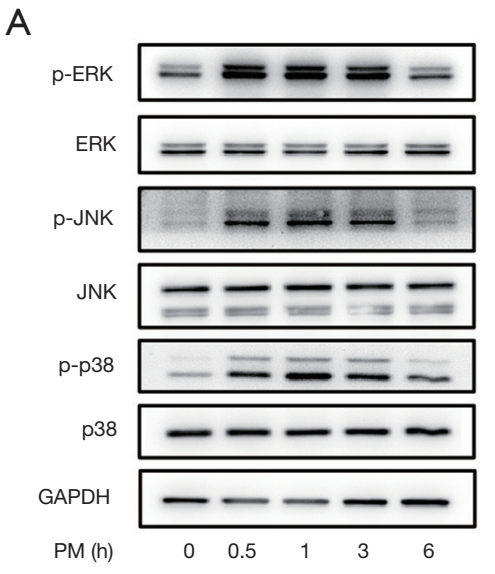

C

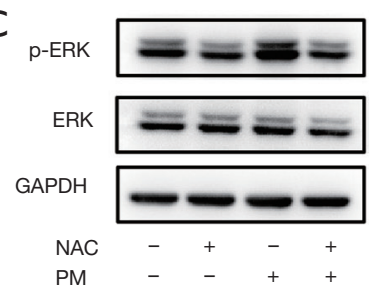

$\mathrm{D}$

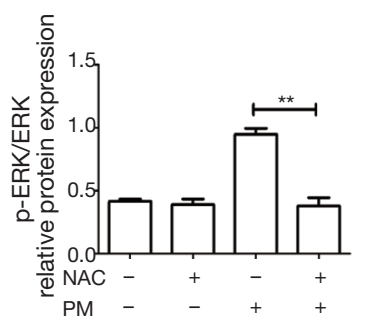

B
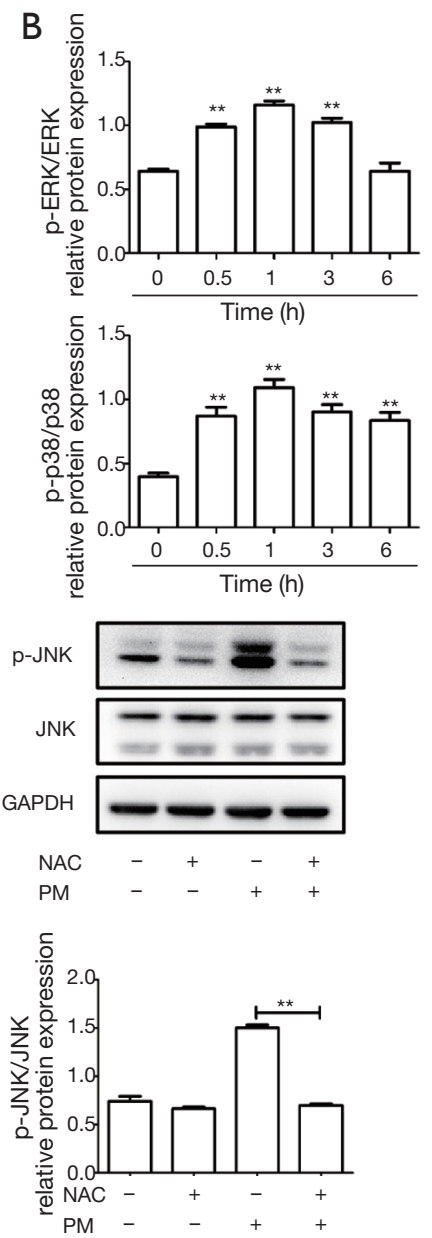
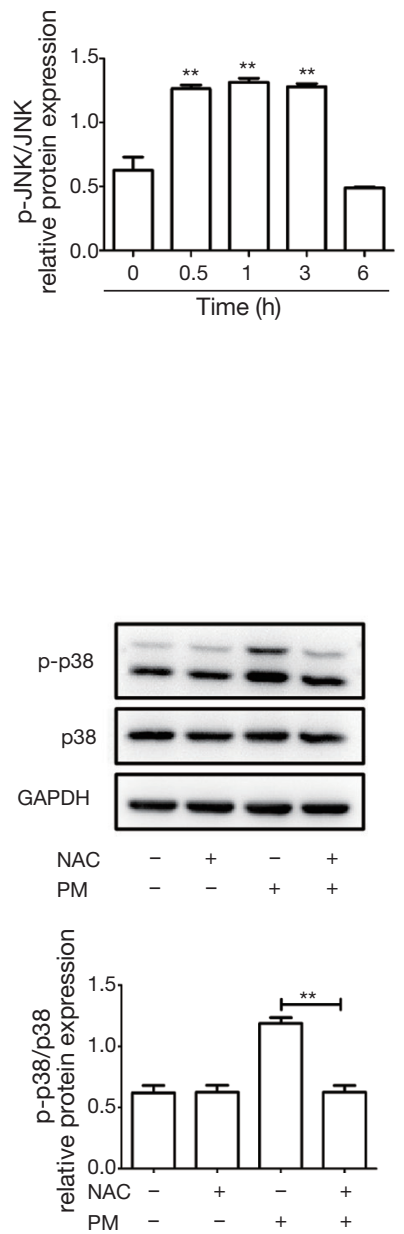

Figure 3 NAC attenuates activation of PM-induced MAPK pathway. (A) HBECs were treated with $300 \mu \mathrm{g} / \mathrm{cm}^{3} \mathrm{PM}$ in a time-dependent $(0.5,1,3$ and $6 \mathrm{~h})$ manner. The phosphorylation of ERK, JNK, and p38 MAPK was detected by western blotting. The optical densities of p-ERK/ERK, p-JNK/JNK and p-p38 MAPK/p38 MAPK bands are shown in (B). Values are the mean \pm SEM; ${ }^{* *}, \mathrm{P}<0.01$, compared with the control group; $\mathrm{n}=3$. (C) HBECs were pretreated with NAC for $30 \mathrm{~min}$ and then stimulated with PM. The activation of ERK, JNK, and p38 MAPK was detected by western blotting. The optical densities of p-ERK/ERK, p-JNK/JNK and p-p38 MAPK/p38 MAPK bands are shown in (D). Values are the mean $\pm \mathrm{SEM}$; ${ }^{* *}, \mathrm{P}<0.01$, compared with the PM group; $\mathrm{n}=3$. NAC, N-acetylcysteine; PM, particulate matter; HBEC, human bronchial epithelial cell.

MMP-9 significantly (Figure 5G). Moreover, expression of the mRNA and protein of COX-2 was inhibited by pretreatment with Bay 11-7082 in HBECs upon PM stimulation significantly (Figure 5 H-7). Collectively, these results demonstrated that the NF- $\mathrm{KB}$ pathway was activated by ROS and MAPK, and was involved in regulating the PM-induced inflammatory response.

\section{PM exposure increases ROS generation in lung tissues}

A mice model of PM-induced acute lung inflammation was established with intratracheal instillation of PM suspension at $100 \mu \mathrm{g} / \mathrm{day} / \mathrm{mouse}$ for two days. NAC was injected (i.p.) before PM exposure each time. The ROS level in lung tissues was detected with DHE staining, MDA activity and $\mathrm{H}_{2} \mathrm{O}_{2}$ assay. DHE staining showed that the ROS level in the bronchiolar and alveolar epithelium was increased significantly after PM exposure, and that NAC pretreatment reduced the PM-induced oxidant stress markedly in lung tissues compared with that in the PM group (Figure 6A,B). As markers of oxidant stress, levels of MDA and $\mathrm{H}_{2} \mathrm{O}_{2}$ in lung tissues were also increased significantly in the $\mathrm{PM}$ 

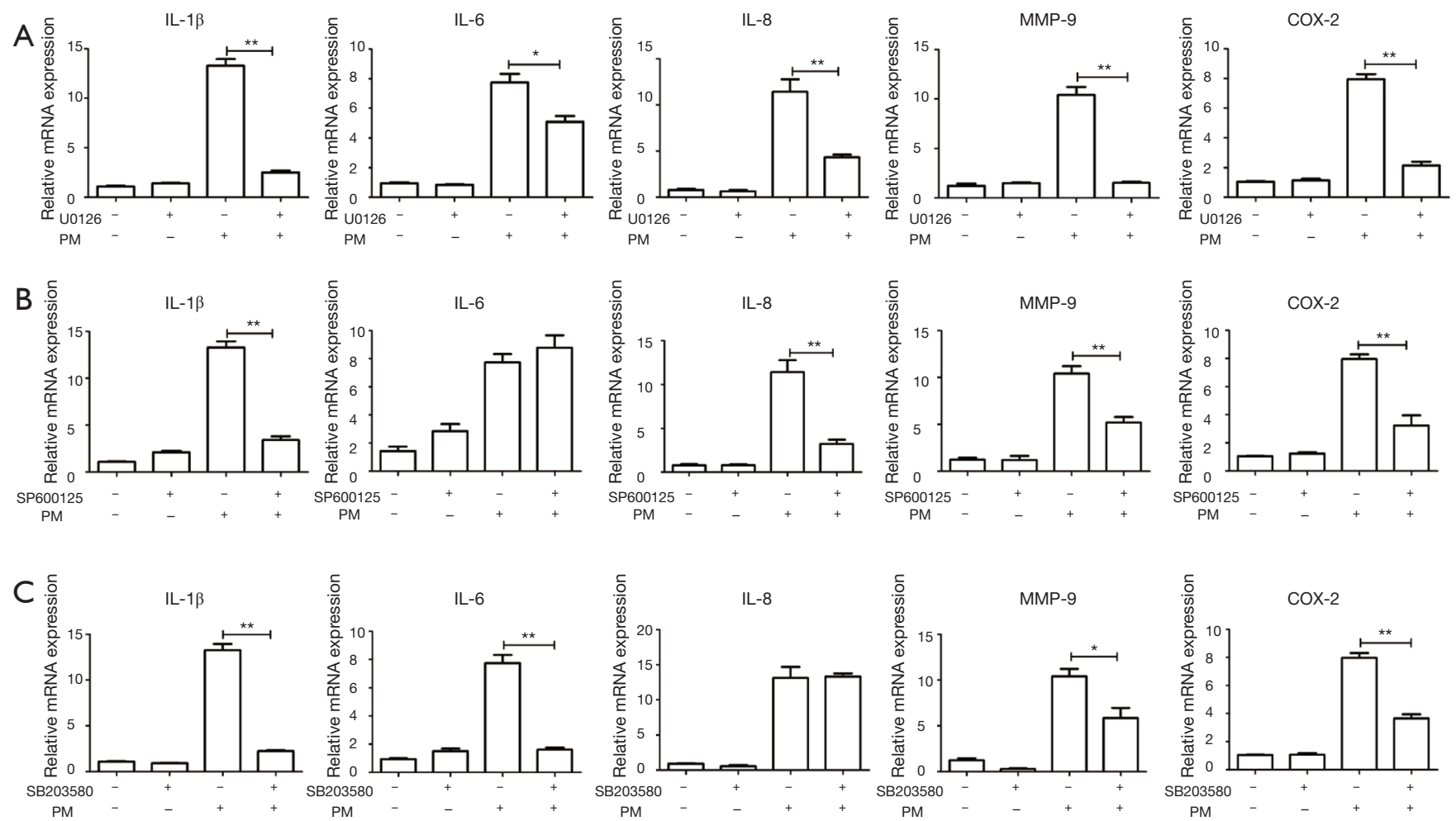

D

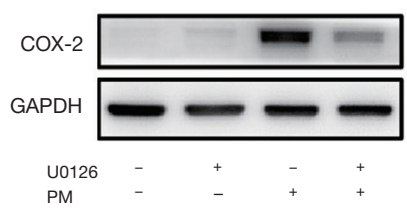

G

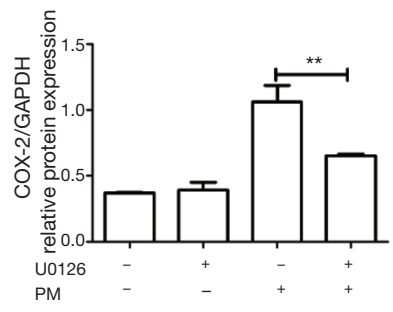

E

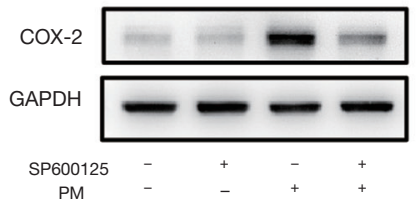

$\mathrm{H}$

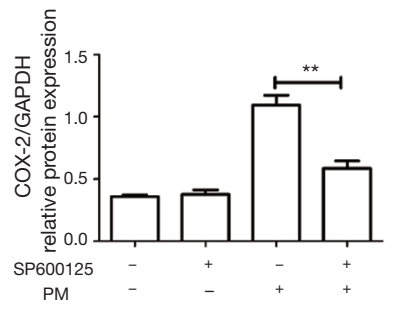

F

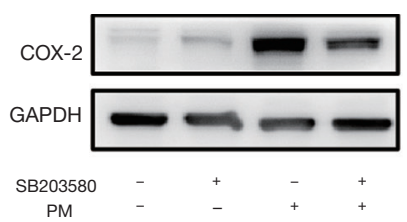

I

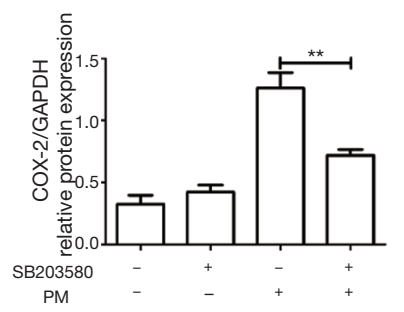

Figure 4 Role of the MAPK pathway in the PM-induced inflammatory response in HBECs. (A) HBECs were pretreated with the ERK inhibitor U0126 (10 $\mu \mathrm{M})$ for $30 \mathrm{~min}$ before PM stimulation. The mRNA expression of IL-1 $\beta$, IL-6, IL-8, MMP-9, and COX-2 was detected by RT-PCR. (B) HBECs were pretreated with the JNK inhibitor SP600125 (10 $\mu$ M) for 30 min before PM stimulation. The mRNA expression of IL-1 $\beta$, IL-6, IL-8, MMP-9, and COX-2 was detected by RT-PCR. (C) HBECs were pretreated with the JNK inhibitor SB203580 $(10 \mu \mathrm{M})$ for $30 \mathrm{~min}$ before PM stimulation. The mRNA expression of IL-1 $\beta$, IL-6, IL-8, MMP-9, and COX-2 was detected by RT-PCR. Values are the mean $\pm \mathrm{SEM}$;, $\mathrm{P}<0.05$ or **, $\mathrm{P}<0.01$, compared with the PM group; $\mathrm{n}=3$. (D) The expression of COX-2 protein upon U0126 pretreatment was detected by western blotting. The optical densities of bands are shown in (G). (E) The expression of COX-2 protein upon SP600125 pretreatment was detected by western blotting. The optical densities of bands are shown in (H). (F) The expression of COX-2 protein upon SB203580 pretreatment was detected by western blotting. The optical densities of bands are shown in (I). Values are the mean \pm SEM; * $\mathrm{P}<0.05$ or ** $\mathrm{P}<0.01$, compared with the $\mathrm{PM}$ group; $\mathrm{n}=3$. HBEC, human bronchial epithelial cell; $\mathrm{PM}$, particulate matter. 

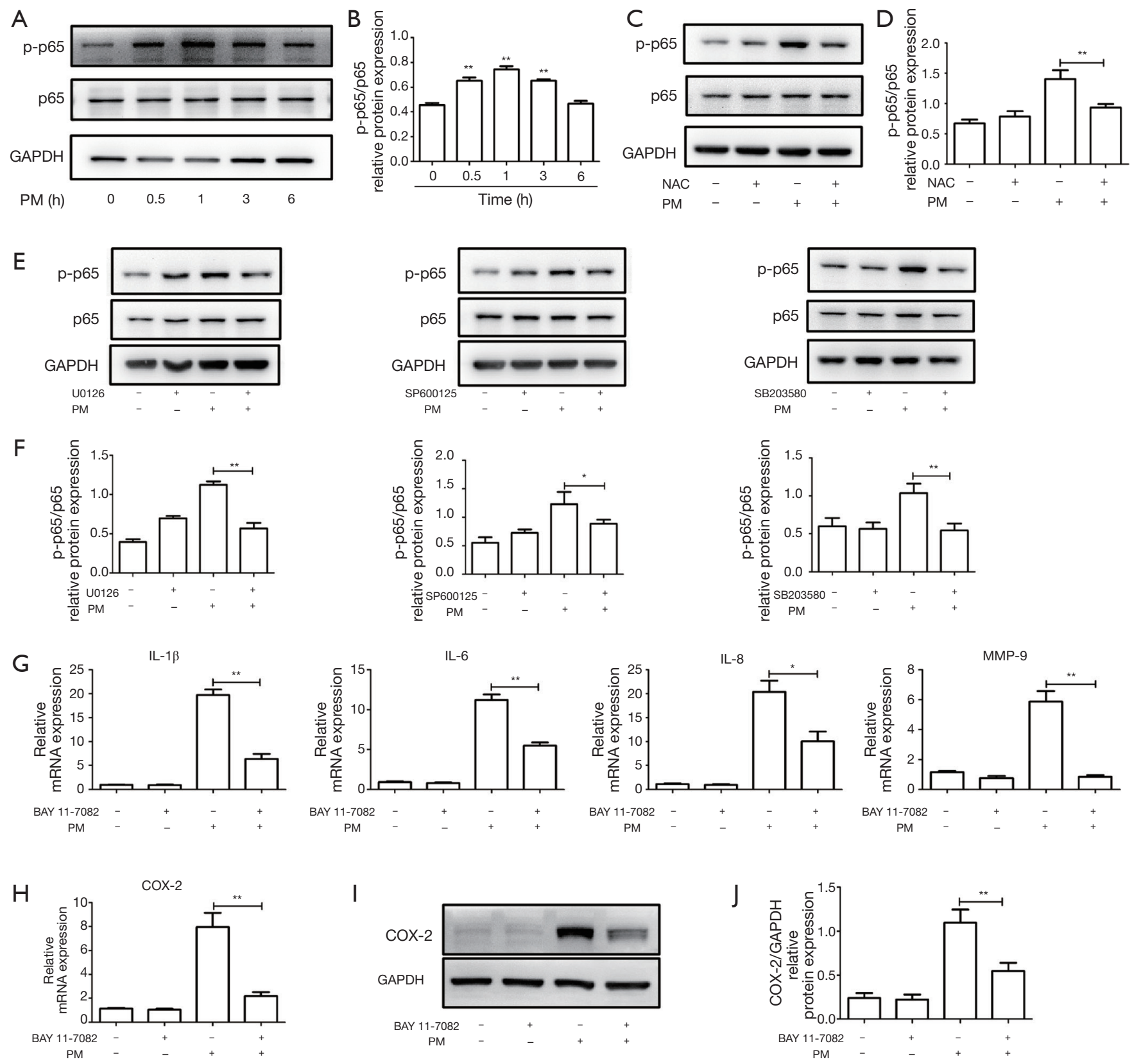

Figure 5 The NAC and MAPK pathway modulate NF- $\kappa B$ activation in the PM-induced inflammatory response in HBECs. (A) HBECs were treated with $300 \mu \mathrm{g} / \mathrm{cm}^{3} \mathrm{PM}$ in a time-dependent $(0.5,1,3$ and $6 \mathrm{~h})$ manner. The phosphorylation of NF- $\mathrm{kB}$ was detected by western blotting. The optical densities of NF- $\kappa B$ bands are shown in (B). Values are the mean $\pm \mathrm{SEM}$; ${ }^{* *}, \mathrm{P}<0.01$, compared with the control group; $\mathrm{n}=3$. (C) HBECs were pretreated with NAC for $30 \mathrm{~min}$ and then stimulated with PM. The activation of NF-kB was detected by western blotting. The optical densities of NF-kB bands are shown in (D). (E) HBECs were pretreated with U0126, SP600125 and SB203580 before $\mathrm{PM}$ treatment, respectively. The activation of NF- $\mathrm{kB}$ was detected by western blotting. The optical densities of NF-kB bands are shown in (F). (G,H) HBECs were pretreated with the NF-kB pathway inhibitor BAY 11-7082 (5 $\mu \mathrm{M})$ before PM treatment. The mRNA expression of IL-1 $\beta$, IL-6, IL-8, MMP-9, and COX-2 was detected by RT-PCR. (I) The expression of COX-2 protein with BAY 11-7082 pretreatment was detected by western blotting; the optical densities of bands are shown in (J). Values are the mean $\pm \mathrm{SEM}$;, $\mathrm{P}<0.05$ or ${ }^{* *}, \mathrm{P}<0.01$, compared with the PM group; n=3. PM, particulate matter; NAC, N-acetylcysteine; HBEC, human bronchial epithelial cell. 


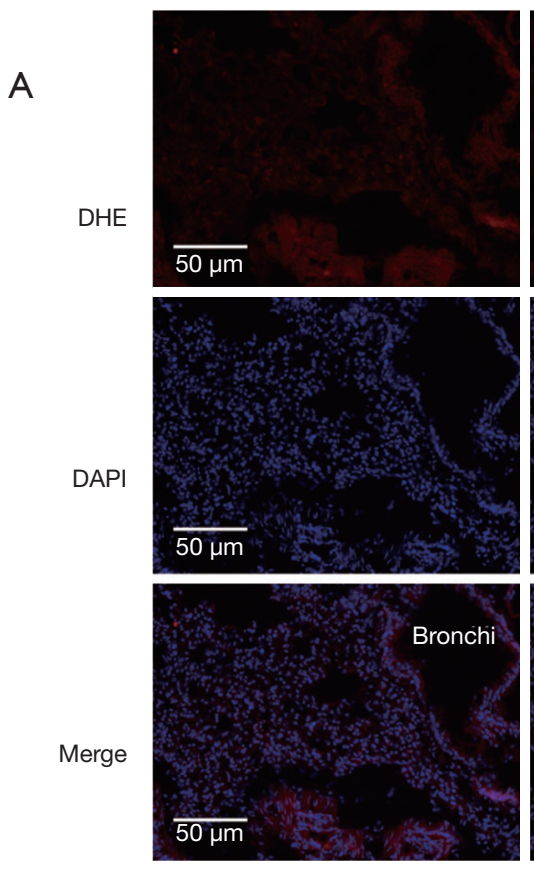

Control

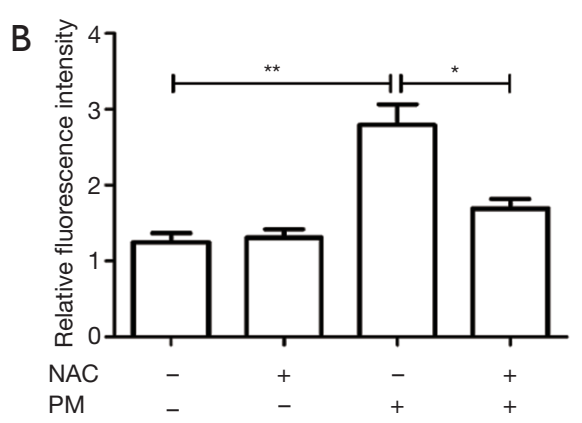

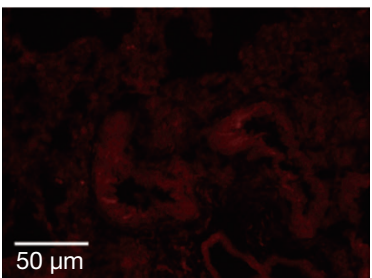
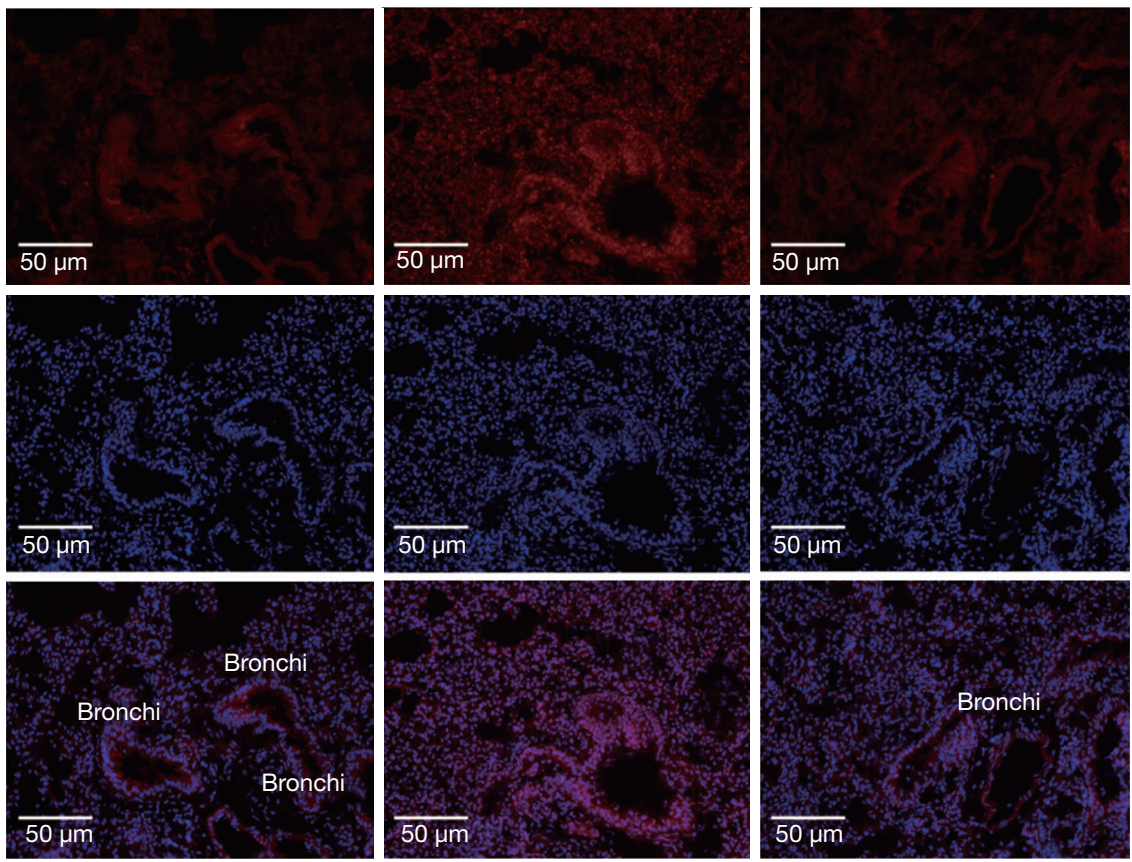

NAC

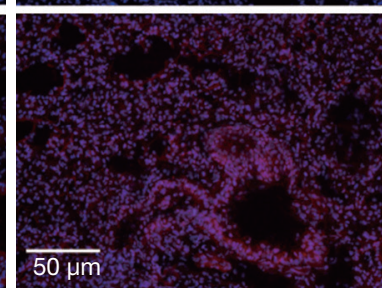

PM

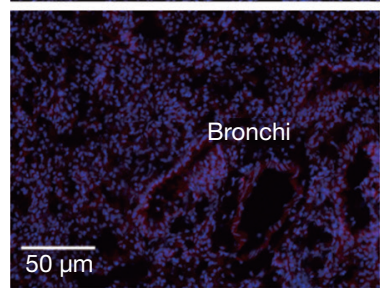

$\mathrm{PM}+\mathrm{NAC}$

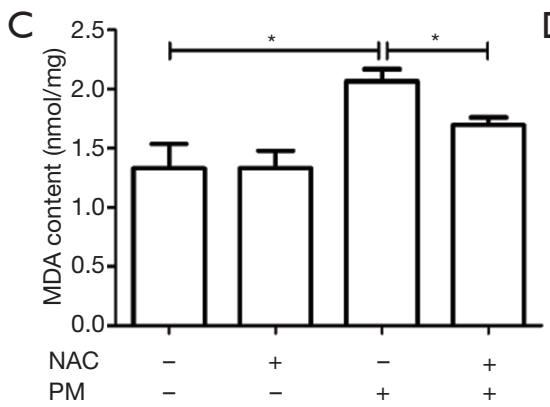

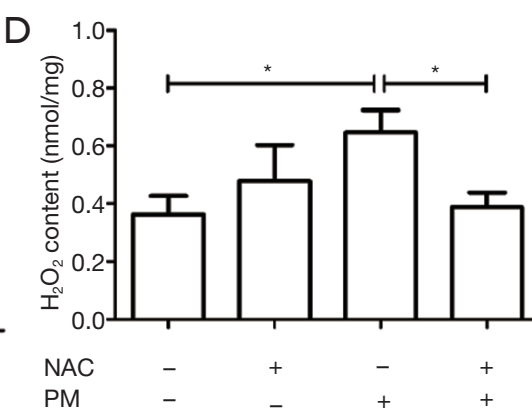

Figure 6 PM exposure increases oxidant stress in lung tissues. Mice were instilled via the intratracheal route with $\mathrm{PM}$ at $100 \mu \mathrm{gg} / \mathrm{day} / \mathrm{mouse}$ for 2 consecutive days. NAC was injected (i.p.) prior to PM exposure each time. (A) The representative images of lung sections stained with DHE. (B) The relative DHE fluorescence intensity in lung tissues from different groups. Values are the mean $\pm \mathrm{SEM}$;, $\mathrm{P}<0.05$ or **, $\mathrm{P}<0.01 ; \mathrm{n}=3$. (C) The MDA activity in lung tissues from different groups was examined. Values are the mean $\pm \mathrm{SEM}$;, $\mathrm{P}<0.05$ or ${ }^{* *}, \mathrm{P}<0.01$; $\mathrm{n}=4$. (D) The $\mathrm{H}_{2} \mathrm{O}_{2}$ generation in lung tissues from different groups was measured. Values are the mean $\pm \mathrm{SEM} ;{ }^{*}, \mathrm{P}<0.05$ or ${ }^{* *}, \mathrm{P}<0.01 ; \mathrm{n}=4$. NAC, N-acetylcysteine; PM, particulate matter.

group compared with those in the control group, and both were inhibited significantly with antioxidant treatment compared with those in the PM group (Figure 6C,D). These results suggested that ROS was generated in lung tissues of PM-treated mice.

\section{NAC attenuates the PM-induced acute lung inflammatory response in mice}

To further demonstrate the pro-inflammatory effect of
$\mathrm{PM}$ on lungs and the role of ROS in the PM-induced lung inflammatory response in vivo, lung histopathology, cell counts, and inflammatory cytokines in BALF were assessed. Histopathologic analyses in lungs showed that PM could obviously induce acute lung inflammatory response in mice. Lung tissues of the PM group exhibited obvious accumulation of inflammatory cells around PM deposits in the airways and alveolar cavity. However, pretreatment with NAC could attenuate the infiltration of inflammatory cells around deposits in lung tissues significantly (Figure $7 A$ ). In 
the PM group, the lung injury score increased significantly compared with that of the control group, but pretreatment with NAC obviously reduced the lung injury score compared with that in the PM group (Figure $7 B$ ). In accordance with these results, the total cell and inflammatory cell numbers in BALF were counted. PM exposure increased the number of total cells and macrophages, especially neutrophils in BALF significantly. Antioxidant treatment reversed the increased number of inflammatory cells in BALF (Figure 7C). In addition, ELISA showed that, compared with the control group, PM exposure in mice notably increased the production of pro-inflammatory cytokines IL-1 $\beta$, IL-6, IL-8 and MMP-9 in BALF, whereas NAC administration could attenuate the secretion of these pro-inflammatory cytokines (Figure 7D). These results suggested that PM exposure could induce acute lung inflammatory response via oxidative stress in vivo.

\section{Discussion}

This study indicated that PM could induce the expression of molecule associated with inflammation and destruction of the extracellular matrix, including IL-1 $\beta$, IL-6, IL-8, and MMP-9. Moreover, the expression of COX-2, an important mediator in inflammation, was increased upon PM exposure. This inflammatory response was demonstrated to be mediated through ROS-dependent activation of ERK, JNK and p38 MAPK signaling pathways and then further activation of the NF-кB signaling pathway (Figure 8). Furthermore, oxidative stress was confirmed to play an important part in our PM-induced acute lung inflammation model and antioxidant treatment reversed the PM-induced inflammatory response in vivo.

Inflammation has been considered to be the central mechanism for the development of various PM-induced pulmonary diseases (30). An array of studies has shown that PM can trigger inflammation response in vivo and in vitro. However, the compositions of PM were often determined to cause different inflammation response. Wang et al. (31) collected ambient PM2.5 from different weather conditions during a sandstorm attack and found a marked association between PM constituents and the inflammatory response. That study suggested endotoxins in PM were positively linked with IL-6 (but not IL-8) production. Moreover, PM components such as silicon and chromium have different effects on IL-6 and IL-8 response. Kurai et al. (32) found that the components of PM2.5 had different biologic effects on airway epithelial cells. PAHs and elemental nickel could induce a higher percentage of apoptosis and IL- $1 \beta$ release whereas iron and chromium had closer associations with DNA damage. Jeong et al. (33) collected PM2.5 samples and obtained two extracts of PM2.5 with different extraction methods, including water-soluble (W-PM2.5) and organic-soluble (O-PM2.5) extracts. A cytokine antibody array showed different expression of pro-inflammation cytokines in human alveolar epithelial cells upon exposure to $\mathrm{W}-\mathrm{PM} 2.5$ or O-PM2.5, respectively. Furthermore, the mice model showed a different response to W-PM2.5 or O-PM2.5, especially with regard to IL-8 expression. Similarly, Kurai et al. (32) found that the compositions of PM could cause different release of IL-5, IL-13, IL-6 and keratinocyte-derived chemokines in asthmatic mice. Our results demonstrated that the urban PM 1649b could induce the release of IL-1 $\beta$, IL- 6 , and IL-8, and increase the expression of MMP-9 which participates in the breakdown of the extracellular matrix and is associated with tissue remodeling in various pulmonary diseases (34). Expression of pro-inflammatory cytokines was also increased significantly in the BALF of PM-exposed mice. COX-2 is a type of pro-inflammatory enzyme and has been reported to be associated with inflammatory response and cytotoxicity upon stimulation with PM from different sources $(27,35,36)$. Here, we found, for the first time, that the urban PM 1649b could induce COX-2 expression in HBECs.

Oxidative stress is the consequence if an excess of ROS is generated while antioxidant defenses are suppressed (37). Several studies have supported the notion that oxidative stress is an important mechanism in the PM-induced inflammatory response, cytotoxicity and carcinogenesis (38). Moreover, oxidative stress is the common cellular response for different components of PM. Metal ions such as zinc, copper and iron have been found to cause redox-reaction dysfunction to generate ROS and to activate downstream signaling pathways to regulate the inflammatory response (18). Organic chemicals such as PAHs, quinones and organic carbon content have strong associations with oxidative stress because they cause mitochondrial injury (39). Our results demonstrated that the urban PM 1649b could increase the generation of ROS in a time- and dose-dependent manner significantly. In addition, 1649b could induce oxidative stress at an early stage in HBECs. For in vivo study, the increase in ROS formation in lung tissues upon PM exposure was detected by DHE staining (DHE can be oxidized to ethidium bromide by superoxide to emit red fluorescence). Meanwhile, the levels of MDA and $\mathrm{H}_{2} \mathrm{O}_{2}$ in lung tissues, as markers of oxidant stress, were increased significantly upon PM 
A
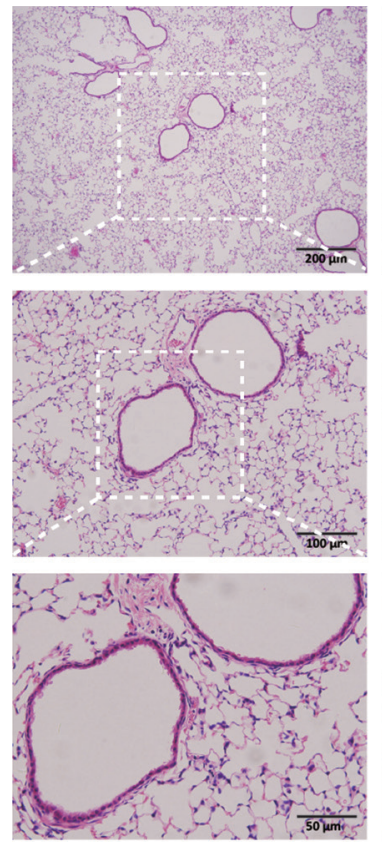

Control
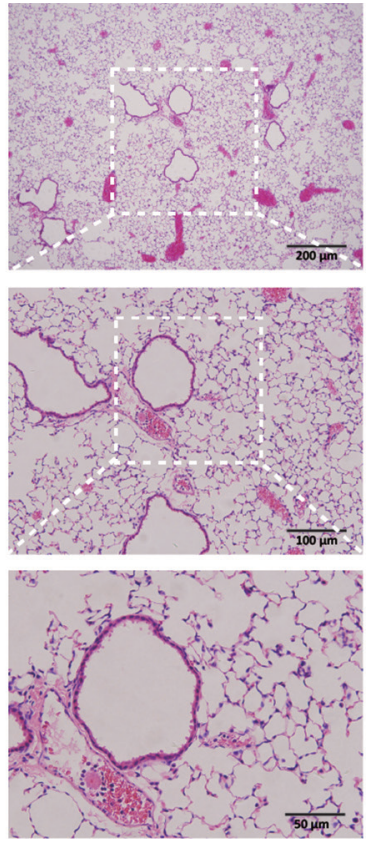

NAC
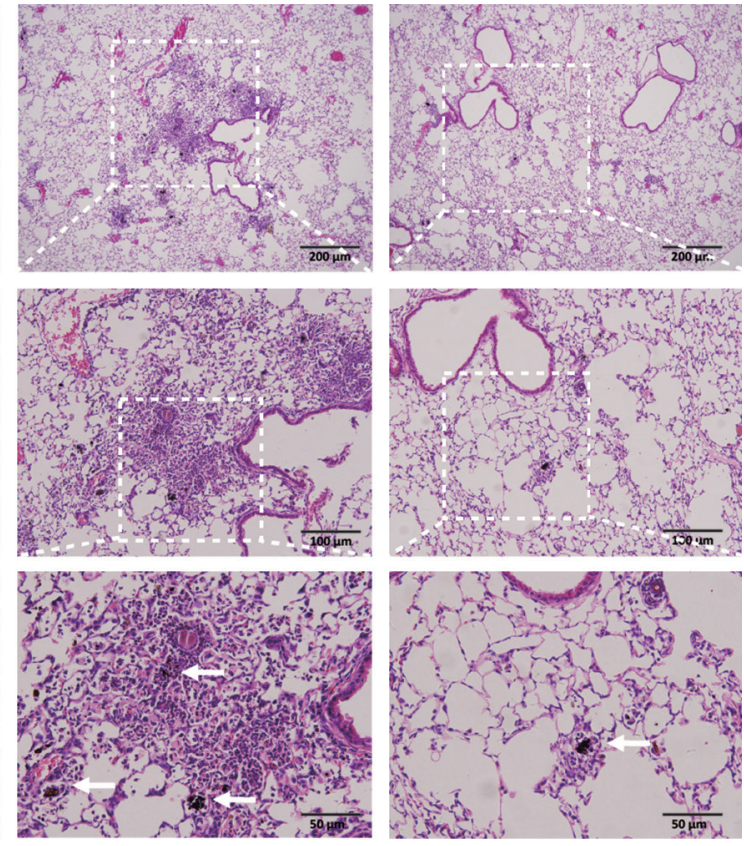

PM

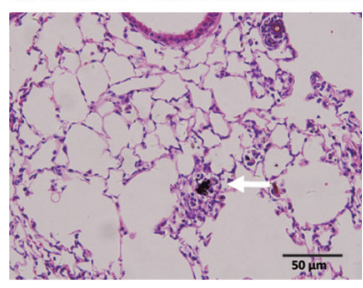

$\mathrm{PM}+\mathrm{NAC}$
B

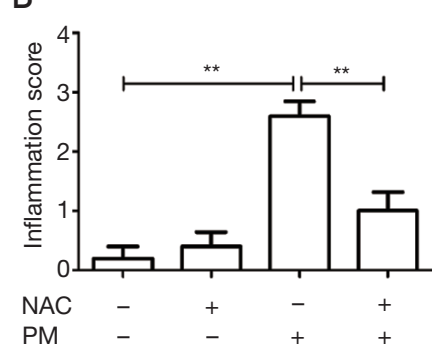

C

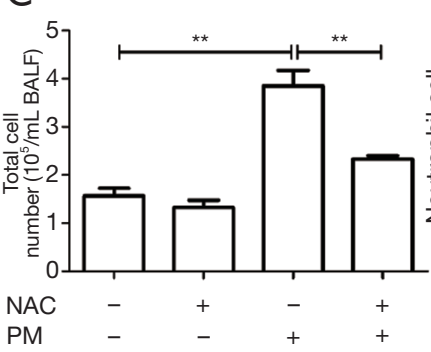

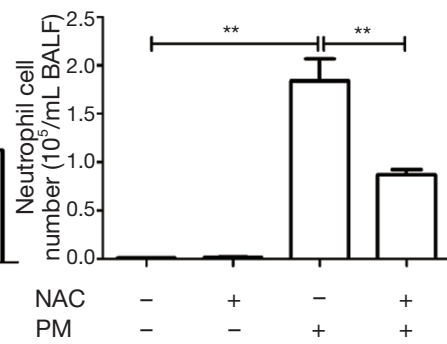
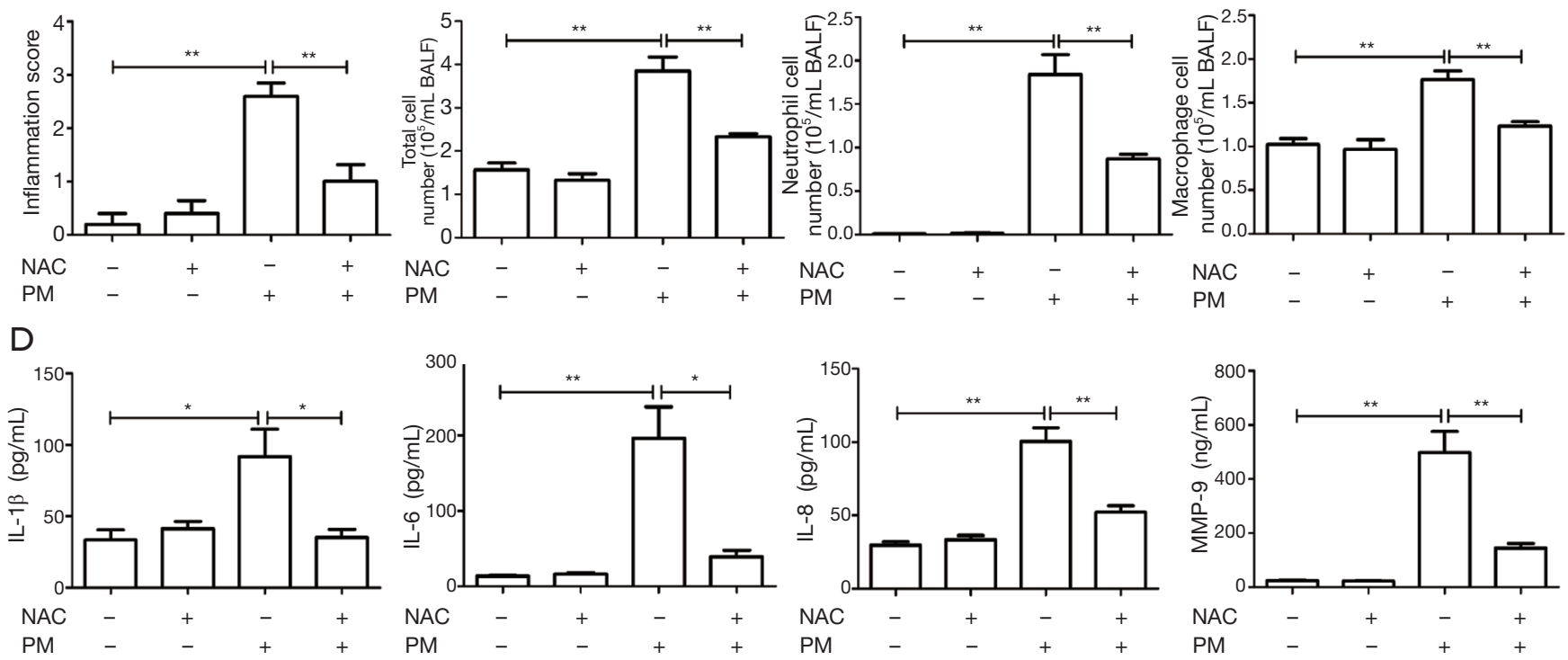

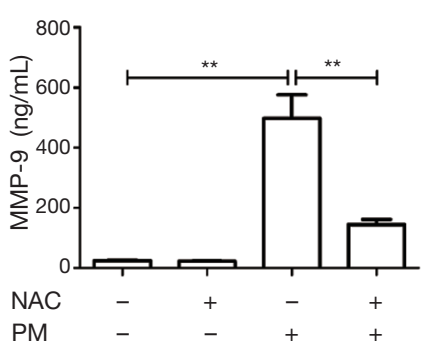

Figure 7 Antioxidant treatment attenuates the PM-induced inflammatory response in vivo. (A) Representative images of lung sections stained with H\&E. (B) The inflammation score for images of lung sections stained with H\&E ( $\mathrm{n}=5$ for each group). (C) The total cell and inflammatory cell numbers in BALF. Values are shown as $10^{5} / \mathrm{mL}$ BALF; $\mathrm{n}=5$. (D) The protein levels of IL-1 $\beta$, IL-6, IL-8 and MMP-9 in the BALF were measured by ELISA. Values are the mean $\pm \mathrm{SEM}$; *, $\mathrm{P}<0.05$ or ${ }^{* *}, \mathrm{P}<0.01 ; \mathrm{n}=5$. NAC, N-acetylcysteine; PM, particulate matter; BALF, bronchoalveolar lavage fluid. 


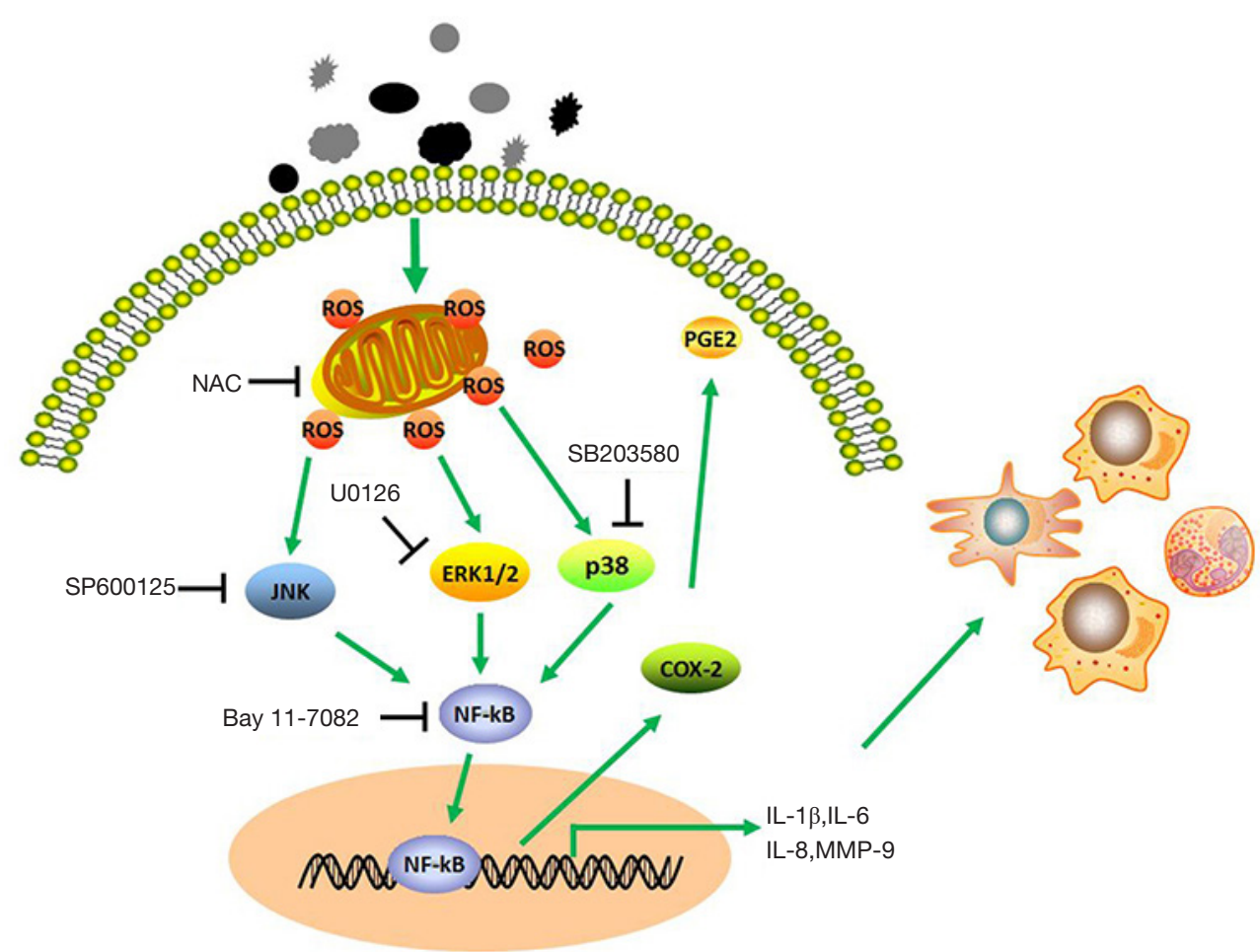

Figure 8 Mechanism of the PM-induced inflammatory response in the lungs (schematic). PM initially triggers oxidative stress to generate ROS and then activates the MAPK pathway. ERK, JNK and p38 MAPK further activate the NF- $\kappa$ B pathway to induce expression of the proinflammatory proteins IL-1 $\beta$, IL-6, IL-8, MMP-9 and COX-2 and infiltration of inflammatory cells in lung tissues. NAC, N-acetylcysteine; $\mathrm{PM}$, particulate matter; ROS, reactive oxygen species.

exposure. NAC has been demonstrated to be an antioxidant that can generate sulfhydryl groups for cells, acting as a ROS scavenger (40). We found that pretreatment with NAC could attenuate the PM-induced inflammatory response markedly in vitro and in vivo. Collectively, oxidative stress is a key modulator and initiator in PM-induced inflammation and could be an ideal target for the prevention and treatment of PM-related diseases.

Accumulated studies have demonstrated that PM can trigger several signaling pathways to regulate the PMinduced inflammatory response, including MAPK and NF$\kappa \mathrm{B}$ signaling pathways (18). The MAPK signaling pathway is one of the most critical intracellular signal-transduction systems, and, in general, is activated by a broad array of extracellular or intracellular stimuli (28). The MAPK pathway is usually activated via a triple kinase cascade: MAPK, MAPK kinase (MAPKK), and MAPKK kinase (MAPKKK). ERK, JNK, and p38 MAPK pathways, as three common members of the MAPK signaling pathway, often participate in different biologic processes in spite of some crosstalk between these signaling pathways at the upper level of cascades (41). In addition, the NF- $\mathrm{BB}$ signaling pathway is a ubiquitous modulator activated by a multitude of stimulants to regulate inflammation, cell cycle, and immune response (42). Rui et al. (43) found that PM2.5 samples could activate ERK, JNK, p38 MAPK and NF-кB pathways. We showed, for the first time, that the urban PM 1649b could also induce the phosphorylation of ERK, JNK, p38 MAPK and NF$\kappa B$. Furthermore, the ERK inhibitor U0126, JNK inhibitor SP600125, p38 MAPK inhibitor SB203580, and NF-кB inhibitor Bay 11-7082 could down-regulate the PM-induced inflammatory response significantly, but the JNK inhibitor SP600125 or p38 MAPK inhibitor SB203580 could not inhibit PM-induced release of IL-6 or IL-8, respectively. These data suggested that PM-induced expression of IL-6 and IL-8 was regulated by different signaling pathways. In addition, the ERK inhibitor U0126, JNK inhibitor SP600125, and p38 MAPK inhibitor SB203580 inhibited activation of the NF- $\kappa B$ signaling pathway upon $\mathrm{PM}$ exposure, suggesting that the PM-induced MAPK signaling 
pathway activated the NF- $\kappa \mathrm{B}$ pathway downstream to promote the inflammatory response.

In conclusion, the present study showed that the urban PM $1649 \mathrm{~b}$ could induce the inflammatory response in vitro and in vivo. This response was regulated by the oxidative stress-mediated MAPK/NF- $\mathrm{BB}$ pathway. Oxidative stress was confirmed to be the key regulator and initiator in PM-related release of pro-inflammatory cytokines and tissue remodeling. These data could contribute to a better understanding of PM-induced adverse health effects.

\section{Acknowledgements}

Funding: This research was supported by the State Key Basic Research Program (973) project (2015CB553404), Doctoral Fund of Ministry of Education of China (20130071110044), Shanghai Science and Technology Committee (15DZ1930600/15DZ1930602), Shanghai Municipal Commission of Health and Family Planning (201540370), National Natural Science Foundation of China (81490533), and National Natural Science Foundation of China (81500026).

\section{Footnote}

Conflicts of Interest: The authors have no conflicts of interest to declare.

Ethical Statement: All protocols for animal experiments were approved by the Animal Care and Use Committee of Zhongshan Hospital, Fudan University.

\section{References}

1. WHO. Global Urban Ambient Air Pollution Database (update 2016). 2016.

2. Huang YC. Outdoor air pollution: a global perspective. J Occup Environ Med 2014;56:S3-7.

3. Guarnieri M, Balmes JR. Outdoor air pollution and asthma. Lancet 2014;383:1581-92.

4. Liu S, Zhou Y, Liu S, et al. Association between exposure to ambient particulate matter and chronic obstructive pulmonary disease: results from a cross-sectional study in China. Thorax 2017;72:788-95.

5. Weichenthal SA, Lavigne E, Evans GJ, et al. Fine particulate matter and emergency room visits for respiratory illness. effect modification by oxidative potential. Am J Respir Crit Care Med 2016;194:577-86.
6. Fajersztajn L, Veras M, Barrozo LV, et al. Air pollution: a potentially modifiable risk factor for lung cancer. Nat Rev Cancer 2013;13:674-8.

7. Bai Y, Sun Q. Fine particulate matter air pollution and atherosclerosis: Mechanistic insights. Biochim Biophys Acta 2016;1860:2863-8.

8. Chung JW, Bang OY, Ahn K, et al. Air pollution is associated with ischemic stroke via cardiogenic embolism. Stroke 2017;48:17-23.

9. Shah AS, Langrish JP, Nair H, et al. Global association of air pollution and heart failure: a systematic review and meta-analysis. Lancet 2013;382:1039-48.

10. Zhang ZM, Whitsel EA, Quibrera PM, et al. Ambient fine particulate matter exposure and myocardial ischemia in the Environmental Epidemiology of Arrhythmogenesis in the Women's Health Initiative (EEAWHI) study. Environ Health Perspect 2009;117:751-6.

11. Girguis MS, Strickland MJ, Hu X, et al. Maternal exposure to traffic-related air pollution and birth defects in Massachusetts. Environ Res 2016;146:1-9.

12. El-Fadel M, Massoud M. Particulate matter in urban areas: health-based economic assessment. Sci Total Environ 2000;257:133-46.

13. Ghio AJ, Soukup JM, Dailey LA. Air pollution particles and iron homeostasis. Biochim Biophys Acta 2016;1860:2816-25.

14. Xu H, Bi XH, Zheng WW, et al. Particulate matter mass and chemical component concentrations over four Chinese cities along the western Pacific coast. Environ Sci Pollut Res Int 2015;22:1940-53.

15. Schlesinger RB. The health impact of common inorganic components of fine particulate matter (PM2.5) in ambient air: a critical review. Inhal Toxicol 2007;19:811-32.

16. Crouse DL, Philip S, van Donkelaar A, et al. A New Method to Jointly Estimate the Mortality Risk of LongTerm Exposure to Fine Particulate Matter and its Components. Sci Rep 2016;6:18916.

17. Zhao Q, Chen H, Yang T, et al. Direct effects of airborne PM2.5 exposure on macrophage polarizations. Biochim Biophys Acta 2016;1860:2835-43.

18. Yan Z, Jin Y, An Z, et al. Inflammatory cell signaling following exposures to particulate matter and ozone. Biochim Biophys Acta 2016;1860:2826-34.

19. Tang W, Du L, Sun W, et al. Maternal exposure to fine particulate air pollution induces epithelial-tomesenchymal transition resulting in postnatal pulmonary dysfunction mediated by transforming growth factor- $\beta /$ Smad3 signaling. Toxicol Lett 2017;267:11-20. 
20. Hamad SH, Schauer JJ, Antkiewicz DS, et al. ROS production and gene expression in alveolar macrophages exposed to $\mathrm{PM}(2.5)$ from Baghdad, Iraq: Seasonal trends and impact of chemical composition. Sci Total Environ 2016;543:739-45.

21. Limón-Pacheco J, Gonsebatt ME. The role of antioxidants and antioxidant-related enzymes in protective responses to environmentally induced oxidative stress. Mutat Res 2009;674:137-47.

22. Chen $\mathrm{ZH}, \mathrm{Wu}$ YF, Wang PL, et al. Autophagy is essential for ultrafine particle-induced inflammation and mucus hyperproduction in airway epithelium. Autophagy 2016;12:297-311.

23. Shimouchi A, Yokota H, Ono S, et al. Neuroprotective effect of water-dispersible hesperetin in retinal ischemia reperfusion injury. Jpn J Ophthalmol 2016;60:51-61.

24. Lee KS, Lee HK, Hayflick JS, et al. Inhibition of phosphoinositide 3-kinase delta attenuates allergic airway inflammation and hyperresponsiveness in murine asthma model. FASEB J 2006;20:455-65.

25. Lagente $\mathrm{V}$, Boichot E. Role of matrix metalloproteinases in the inflammatory process of respiratory diseases. J Mol Cell Cardiol 2010;48:440-4.

26. Iezzi A, Ferri C, Mezzetti A, et al. COX-2: friend or foe? Curr Pharm Des 2007;13:1715-21.

27. Zhao Y, Usatyuk PV, Gorshkova IA, et al. Regulation of COX-2 expression and IL-6 release by particulate matter in airway epithelial cells. Am J Respir Cell Mol Biol 2009;40:19-30.

28. Sun J, Nan G. The Mitogen-activated protein kinase (MAPK) signaling pathway as a discovery target in stroke. J Mol Neurosci 2016;59:90-8.

29. Herrington FD, Carmody RJ, Goodyear CS. Modulation of NF-kappaB signaling as a therapeutic target in autoimmunity. J Biomol Screen 2016;21:223-42.

30. Kelly FJ, Fussell JC. Air pollution and airway disease. Clin Exp Allergy 2011;41:1059-71.

31. Wang B, Li N, Deng F, et al. Human bronchial epithelial cell injuries induced by fine particulate matter from sandstorm and non-sandstorm periods: Association with particle constituents. J Environ Sci (China) 2016;47:201-10.
32. Kurai J, Watanabe M, Sano H, et al. The effect of seasonal variations in airborne particulate matter on asthma-related airway inflammation in mice. Int J Environ Res Public Health 2016;13. pii:E579

33. Jeong SC, Cho Y, Song MK, et al. Epidermal growth factor receptor (EGFR)-MAPK-nuclear factor(NF)-кB-IL8: A possible mechanism of particulate matter(PM) 2.5-induced lung toxicity. Environ Toxicol 2017;32:1628-36.

34. Atkinson JJ, Senior RM. Matrix metalloproteinase-9 in lung remodeling. Am J Respir Cell Mol Biol 2003;28:12-24.

35. Vogel CF, Sciullo E, Wong P, et al. Induction of proinflammatory cytokines and C-reactive protein in human macrophage cell line U937 exposed to air pollution particulates. Environ Health Perspect 2005;113:1536-41.

36. Bai N, Tranfield EM, Kavanagh TJ, et al. Exposure to diesel exhaust upregulates COX-2 expression in ApoE knockout mice. Inhal Toxicol 2012;24:518-27.

37. Brigelius-Flohé R. Commentary: oxidative stress reconsidered. Genes Nutr 2009;4:161-3.

38. Øvrevik J, Refsnes M, Låg M, et al. Triggering mechanisms and inflammatory effects of combustion exhaust particles with implication for carcinogenesis. Basic Clin Pharmacol Toxicol 2017;121:55-62.

39. Li N, Xia T, Nel AE. The role of oxidative stress in ambient particulate matter-induced lung diseases and its implications in the toxicity of engineered nanoparticles. Free Radic Biol Med 2008;44:1689-99.

40. Shimizu MH, Gois PH, Volpini RA, et al. N-acetylcysteine protects against star fruit-induced acute kidney injury. Ren Fail 2017;39:193-202.

41. Acosta AM, Kadkol SS. Mitogen-activated protein kinase signaling pathway in cutaneous melanoma: an updated review. Arch Pathol Lab Med 2016;140:1290-6.

42. Panday A, Inda ME, Bagam $P$, et al. Transcription factor NF- $\kappa \mathrm{B}$ : an update on intervention strategies. Arch Immunol Ther Exp (Warsz) 2016;64:463-83.

43. Rui W, Guan L, Zhang F, et al. PM2.5-induced oxidative stress increases adhesion molecules expression in human endothelial cells through the ERK/AKT/NF- $\mathrm{B}$ dependent pathway. J Appl Toxicol 2016;36:48-59.
Cite this article as: Wang J, Huang J, Wang L, Chen C, Yang D, Jin M, Bai C, Song Y. Urban particulate matter triggers lung inflammation via the ROS-MAPK-NF- $\kappa \mathrm{B}$ signaling pathway. J Thorac Dis 2017;9(11):4398-4412. doi: 10.21037/ jtd.2017.09.135 